\title{
Lumen
}

Selected Proceedings from the Canadian Society for Eighteenth-Century Studies

\section{L’écrivain rival de ses personnages : Marivaux, La Voiture embourbée}

\section{Raymond Joly}

Volume 23, 2004

URI : https://id.erudit.org/iderudit/1012195ar

DOI : https://doi.org/10.7202/1012195ar

Aller au sommaire du numéro

Éditeur(s)

Canadian Society for Eighteenth-Century Studies / Société canadienne d'étude du dix-huitième siècle

ISSN

1209-3696 (imprimé)

1927-8284 (numérique)

Découvrir la revue

Citer cet article

Joly, R. (2004). L'écrivain rival de ses personnages : Marivaux, La Voiture embourbée. Lumen, 23, 205-219. https://doi.org/10.7202/1012195ar

Copyright (C Canadian Society for Eighteenth-Century Studies / Sociéte canadienne d'étude du dix-huitième siècle, 2004
Ce document est protégé par la loi sur le droit d'auteur. L'utilisation des services d'Érudit (y compris la reproduction) est assujettie à sa politique d'utilisation que vous pouvez consulter en ligne.

https://apropos.erudit.org/fr/usagers/politique-dutilisation/ 


\title{
11. L'écrivain rival de ses personnages : Marivaux, La Voiture embourbée
}

\author{
À Françoise Rubellin
}

Nulle mémoire humaine n'est d'une capacité infinie. Ce n'est donc pas faire outrage au lecteur de lui rappeler que La Voiture embourbée, parue sans nom d'auteur à la fin de 1713 ou au début de 1714, raconte comment le «carrosse de voiture» qui assure la liaison avec Nemours, parti un jour d'hiver, juste après le carnaval, avec à son bord cinq passagers dont le narrateur, fut victime à la nuit tombante de l'accident qui donne son titre au livre. Les voyageurs durent patauger dans les ronces et la boue avant de trouver une misérable auberge où ils furent contraints d'attendre le jour et la remise en état du véhicule. Ils purent Dieu merci améliorer quelque peu la pitance que leur offrait leur hôtesse grâce à la générosité rémunérée du curé du village, auprès de qui deux d'entre eux s'étaient détachés en ambassade. Pour se distraire en attendant de reprendre la route, le narrateur proposa aux rescapés, auxquels s'était joint le neveu du curé, d'improviser à tour de rôle une histoire, ce «Roman impromptu» qui occupe les trois quarts du livre ${ }^{1}$.

Entre le début et la fin de La Voiture embourbée, finalement, il ne s'est guère passé que ce que Jean Bellemin-Noël appellerait du racontage. Les premiers mots sont : «Enfin, mon cher, je vous tiens parole, voici le récit de la petite histoire que je vous avais promise» (p. 317), et les derniers : «j'arrivai à Nemours, je quittai mes voyageurs, et je fis

1 Je renvoie à l'édition procurée par Frédéric Deloffre dans les CEuvres de jeunesse de Marivaux, Paris, Gallimard, coll. «Bibliothèque de la Pléiade», 1972. Le texte a été contrôlé sur un microfilm de l'édition originale. Je modernise l'orthographe comme la Pléiade, sans toucher toutefois aux accords, mais j'ai parfois choisi des partis différents en matière de ponctuation. Voir Raymond Joly, «De la ponctuation, à propos de Marivaux», Lumen, 20, 2001, p. 123-135. 
résolution de vous faire le récit de nos plaisirs ; vous me le fites promettre, ma parole est acquittée, serviteur» (p. 388). Le locuteur avait décidé de faire un récit à son ami («je fis résolution»), il s'est arrangé pour que son désir lui revienne sous la forme d'une demande de l'autre ("vous me le fites promettre»). Le plaisir de la parole s'est trouvé légitimé par le fait qu'elle tenait parole à un destinataire en droit de l'exiger, le dénommé «Mon cher». Mais celui-ci, bien sûr, n'est qu'une utilité : à peine a-t-il été interpellé qu'apparaissent derrière lui "ceux qui liront ceci», c'est-à-dire l'image du public, des lecteurs concrets virtuels, derrière lesquels se profile le destinataire idéal, celui qui comprendra l'auteur comme il veut être compris et l'aimera en conséquence. Derrière tous ceux-là enfin (mais cette spatialisation est bien défectueuse, car c'est au cœur même de la production textuelle dont ils sont le moteur que logent tous ces êtres sans corps) : le grand Autre, ce qui nous constitue comme sujets contraints à parler et détient l'aune à laquelle se mesure notre parole.

Mais le racontage se déploie à plus d'un niveau, et avec une certaine ambiguité, puisqu'il s'agit, si nous en croyons la première phrase, du "récit de la petite histoire que je vous avais promise». Mais quelle histoire ? La chose serait simple si nous en restions à l'Avis du Libraire : la "petite histoire» qu'il offre au public se ramène au «récit de l'aventure plaisante arrivée dans une voiture, il y a quelque temps, à l'auteur et à quatre autres personnes» (p. 311). Le narrateur, lui, nous entraîne sur une autre piste : «Ce récit sera fidèle, et je vous le donne tel que je l'ai entendu faire, et tel que je l'ai fait moi-même ; car vous savez que j'étais du nombre de ceux qui l'ont récité». Il faut se souvenir que réciter, à l'époque, ne signifiait pas seulement «Dire à haute voix ce qu'on sait de mémoire», mais précisément «faire un récit» : «Raconter, faire une relation de quelque chose». Ce que notre narrateur avait promis, c'était donc le récit d'un récit, la transcription du roman impromptu "récité» par les voyageurs au fur et à mesure qu'ils l'inventaient dans la calamiteuse auberge.

N'empêche qu'achevant le premier quart du livre sans qu'aient encore débuté ladite histoire ni ledit récit, le narrateur se fait des scrupules : «Peut-être, mon cher, aurez-vous trouvé trop long le sujet qui conduit à notre histoire, mais le sujet est une petite histoire aussi ; et comme je n'ai eu dessein que de vous divertir, peu m'a dû importer que ce soit, ou par le sujet, ou par l'histoire» (p. 334-335). On voit qu'il ne faut pas entendre ici par sujet «la matiere qu' on traitte, l'evenement qu'on raconte», pour parler comme Furetière et comme nous le faisons encore lorsque nous disons résumer le sujet d'un livre ou d'un film. Le sujet à quoi pense notre narrateur se définit par "Cause, occasion, fondement», comme dans "J'ay pris sujet de vous escrire, pour dire, 
l'occasion $^{2} »$. C'est justement ce que le narrateur expliquait dans son exorde : "pour donner à ceux qui liront ceci raison des goûts différents dont cette histoire sera écrite, je vais commencer par les choses qui l'ont occasionnée ${ }^{3} »$ (p. 317). Voilà donc deux petites histoires dont l'une expose le sujet de l'autre, c'est-à-dire raconte ce qui a amené à raconter l'autre.

Si la voiture embourbée fut le «sujet»-occasion-cause du roman impromptu que "réciteront» les trois derniers quarts de La Voiture embourbée, je voudrais dans ces quelques pages laisser entrevoir le «sujet»-occasion-cause qui a pu amener son auteur à en écrire les quatre quarts, et d'abord le premier. Il s'agit dans tout cela de plaire à deux belles dames, à une petite société que menacent l'ennui et l'inanition au cours d'une interminable veille, à l'ami qui reçoit prétendument la relation de l'aventure, et au public lecteur. Ils sont plusieurs à s'y employer : l'auteur lui-même, avantageusement connu grâce à son précédent roman, nous dit l'Avis du libraire, et qui a besoin de manger lui aussi ; un narrateur qui joue un rôle capital dans l'histoire qu'il raconte ; trois autres hommes dont deux se piquent d'esprit, et jusqu'aux deux dames. Sur la route fictive de Nemours, en ce premier jeudi de carême, les enjeux ne manquent pas pour faire éclater des rivalités inexpiables. L'échauffourée est assez violente pour que la narratologie $\mathrm{y}$ perde son bonnet. Comme dans ces tourbillons de poussière où les héros de L'Iliade ne savent plus s'ils combattent encore entre eux ou s'attaquent aux immortels, on n'aperçoit ici que horions et moulinets,

2 Furetière toujours - Dans la préface de la Voiture, un auteur impute aux instances de ses amis la publication d'un ouvrage de valeur douteuse ; impitoyable, le critique rétorque : «n'aviez-vous pas le secours du feu, qui pouvait faire évanouir le mauvais sujet de leurs importunités ?» (p. 314.) L'Apicius des Dialogues des morts de Fontenelle part à la recherche d'un certain poisson, ce qui fait de ce poisson le sujet du voyage. Cet usage s'est considérablement restreint. Le Trésor de la langue française relève toujours sujet dans l'acception de "Motif, raison", mais non plus d' «occasion", et prend soin de préciser : «Souvent dans un contexte à connotation défavorable»; on $a$ sujet de s'inquiéter ou de se plaindre, il n'est pas normal de rire et de pleurer sans sujet, etc.

3 Françoise Rubellin observe très justement qu'on aurait pu attendre «les goûts différents dont ce roman a été improvisé» plutôt que "dont cette histoire sera écrite» («Folie narrative et raison romanesque dans La Voiture embourbée de Marivaux», dans René Démoris et Henri Lafon (édit.), Folies romanesques au siècle des Lumières, Paris, Desjonquères, 1998, p. 65-81) : "c'est privilégier la création plutôt que la retransmission, et valoriser l'unité du roman» (p. 77). 
sans bien distinguer si le terrain est l'auberge imaginaire, le milieu des gens de lettres ou l'âme de l'auteur.

Le narrateur, s'adressant à son ami, lui écrivait : «je fis résolution de vous faire le récit de nos plaisirs». Ils sont bien modestes et la liste n'en est pas longue : «la fatigue des veilles et des plaisirs» du carnaval (p. 317), qui a fait dormir notre homme toute la matinée au départ du carrosse et qui ne mérite que ces sept mots ${ }^{4}$; ensuite la prise de tabac au réveil, la causerie devant le feu après le bon repas qui a précédé l'accident, le feu encore dans la misérable auberge, le vin exquis du curé et le «morceau de beurre très frais» qui accompagna sa «stockfiche [morue], aussi bonne que de la stockfiche le peut être» (p. 331). C'est peu de chose. Quel ami se serait fait promettre la relation de cela ? Mais il y a le racontage, le plaisir principal, qui console de l'ennui et de l'inconfort d'être cloué dans un bouge, qui console peut-être de porter de plus lourdes entraves.

Car je veux bien qu'on tire un livre d'être parti pour Nemours et d'y être arrivé, mais traditionnellement l'auteur aurait fait en sorte qu'entre la situation initiale et la situation terminale l'état des personnages se fût quelque peu transformé. En particulier, «l'amour faisant le sujet d'un roman", principe indiscutable que rappelle l'un des conteurs dans celuici (p. 324), on voudrait savoir ce qui est advenu, sous ce rapport, du personnage qui s'est attribué la position dominante dans le récit, le narrateur. Et, puisqu'il est tellement question de raconter, la position des conteurs les uns par rapport aux autres et face à leur auditoire devient inévitablement l'enjeu d'une rivalité. Or, sous ces deux aspects, notre narrateur est pris dans un tel enchevêtrement de contradictions et $d^{\prime}$ 'ambivalences qu'il ne lui reste aucune possibilité de mouvement.

En fait, embourbé ne décrirait pas mal sa situation, et nous aurions tort de passer trop vite sur l'incident trivial qui est le «sujet» de tout le reste. "Je ne vous ferai point un détail exact de $1 a^{5}$ conversation de notre après-dînée : tout cela ne fait rien à notre histoire», lisons-nous, et c'est un mensonge ; «qu'il vous suffise de savoir que la tendresse et l'amour furent les sujets que nous traitâmes» (p. 318). Et voici que, plus loin,

4 Mais le livre entier arbore des traits carnavalesques (Rubellin, «Folie narrative», p. 78).

5 Et non «notre», dittographie des CEuvres de jeunesse. 
La conversation sur l'amour était fort échauffée quand, par l'imprudence des cochers, qui vidaient derrière nous une bouteille de grès, nos chevaux sans guides enfilèrent un chemin plein d'un limon gras où les malheureux animaux s'enfoncèrent, aussi bien que les roues de la pesante voiture, qui resta comme immobile (p. 320).

Il est commode d'imputer l'accident à l'inattention des cochers pompettes, mais ils n'étaient pas les seuls à céder à l'ivresse: «La conversation sur l'amour était fort échauffée». La brutalité et l'acharnement dominent dans la scène qui se déroule ensuite : les coupables, voyant l'effet de leur négligence,

s'approchèrent, avec des dia, hue, et maints claquements de fouet ; les chevaux avertis s'efforcent, suent, et se renfoncent davantage ; les cochers épuisent, enrouent leur altéré gosier, fouettent comme des charretiers : inutiles efforts, déjà les chevaux soufflent, reniflent ; nos phaétons jurent, et rien ne s'avance. Nous descendons de carrosse ; ils redoublent et les coups et les jurements, et la Bastille $n^{\prime}$ est pas plus ferme sur ses fondements que nos roues le sont sur la funeste boue ${ }^{6}$ (p. 320).

La comparaison de la fin semble banale : nous l'apprécions autrement lorsque, dans le «Roman impromptu», Bastille est devenu le nom d'une jeune fille particulièrement exquise, qui finit ligotée dans une caverne où un sadique se livre à d'effrayants excès (p. 358ss). Les voyageurs prennent finalement le parti de marcher en direction des quelques chaumières qu'ils aperçoivent au loin, et le narrateur conclut : «Quelle chute, grands dieux ! de la conversation la plus aimable à cette triste extrémité ! Amour, Amour, voilà ton portrait, tu nous séduis par de doux commencements, mais toujours d'affreuses catastrophes sont le nœud des appâts flatteurs dont tu nous as trompé» (p. 321). Ainsi, c'est l'amour qui a précipité la voiture dans le limon gras, et ce n'est pas moi qui le dis. Cela rappelle irrésistiblement ce passage de Pharsamon (que Marivaux écrivait exactement à la même époque) où un gentilhomme, apercevant le héros évanoui au milieu des flaques de graisse dans une cuisine où vient de se dérouler un combat avilissant, déclare en s'adressant aux dames : «C'est apparemment quelque honnête homme à qui l'amour a tourné la cervelle [...], et voilà dans quel état vous jetez les cavaliers ${ }^{7} »$.

6 Il y aurait lieu de prêter attention à la fréquence $\operatorname{des} f$. Autre cas à la p. 318 .

7 Pharsamon, dans CEuvres de jeunesse, p. 586. Ce passage est évoqué dans Joly, Le «Pharsamon» de Marivaux ou Comment s'inventer un sexe, Paris, P.U.F., coll. «Le texte 
«Pardon, mon cher [poursuit ici notre voyageur], si j'interromps ma narration par cette parenthèse ; mais notre situation alors était si triste, que le simple portrait que j'en fais $\mathrm{m}^{\prime} \mathrm{en}$ inspire encore des réflexions mélancoliques $^{8} »($ p. 321).

Périlleux sont les chemins de l'amour pour celui à qui l'auteur fait écrire ces lignes! Dès qu'il en est question, la jouissance s'avère impossible à maîtriser, les têtes s'échauffent, le pauvre garçon est assailli par des fantasmes de violence et d'abjection qui en se retirant le laissent dans un état dépressif et mélancolique. Il est normal, dirais-je, qu'il ait affaire dans une ville dont le nom ne tolère la résonance d'amours que préalablement étouffée par la marque de la négation - et cela avant même qu'on n'invoque La Princesse de Clèves.

Toujours est-il que le carrosse de Nemours met notre héros face à face avec un personnage qui a toujours exercé sur son créateur une vive fascination - au sens strict, mélange d'horreur et d'invincible attrait. Je veux parler de la «dame passablement belle, ni jeune ni âgée, mais assez raisonnablement l'un et l'autre pour justifier l'amour ou l'indifférence qu'on aurait eu pour elle» (p. 317). Elle est redoutable en effet : en plus de sa beauté, de son air et de son geste doux, d'un naturel tendre, d'une élégance et d'une délicatesse de manières sans défaut, elle est douée de «beaucoup d'esprit» (p. 321). On admettra qu'il y a de quoi avoir peur, puisque se laisser attirer par de tels charmes revient à se jeter dans les bras de la mère idéale et que la mère idéale n'attend que le moment propice pour révéler sa vraie nature, castratrice et meurtrière. Les productions de Marivaux contemporaines de notre roman, Pharsamon et Les Effets surprenants de la sympathie, nous la montrent à l'œuvre : incapable $\mathrm{d}$ 'assumer son sexe, elle travaille énergiquement à bloquer autour d'elle tout accès à la virilité. Notre narrateur passe donc à l'offensive : dans le récit en lardant de perfidies tous les endroits où il parle de sa compagne de voyage, dans la fiction en la persiflant et en l'agressant ; c'est une muflerie en effet (elle le relèvera discrètement, p. 352, 354-355) que de lancer l'improvisation collective par une parodie des romans dont elle fait tant de cas.

rêve», 1995, p. 119. Je renvoie à l'ensemble du dernier chapitre (p. 103-122) pour ce qui regarde le rapport entre le narrateur et les figures féminines de La Voiture embourbée.

8 On attendrait «m'inspire» et non «m'en inspire». Le second en s'explique par une banale dittographie, de Marivaux ou du typographe, ou par l'interférence d'une autre construction dans l'esprit de l'auteur : notre situation était si triste qu'en en faisant le simple portrait, il m'en vient encore des réflexions mélancoliques. 
Notons qu'elle n'est guère mieux traitée par sa propre fille quand le tour vient à celle-ci de poursuivre le roman impromptu. La dame, relançant le dé après le narrateur, avait travesti son hérö̈ne en chevalier et l'avait introduite dans une caverne où régnait un cruel magicien; le troisième conteur, le bel esprit, avait renchéri par des orgies d'atrocités. La demoiselle de quinze ans se fait gloire de dissiper d'un mot tous ces enchantements. Elle commence par les énumérer en remontant jusqu'à la caverne de sa mère, qu'elle s'empresse de «détruire» et de «boucher pour jamais» (p. 378-379), ce qu'il n'est pas nécessaire d'avoir beaucoup pratiqué La Philosophie dans le boudoir, je pense, pour lire en éprouvant quelque malaise. Ensuite, l'héroïne que la dame avait dotée d'un courage martial, elle la ramène à la condition d'une pauvre femme craintive errant la nuit dans la campagne avec de burlesques habits d'homme et le pronom personnel masculin comme seuls vestiges de son avatar guerrier.

Au surplus, il importe de souligner que l'attrait de la figure maternelle ne réside pas seulement dans ce qu'elle représente comme satisfactions possibles en qualité d'objet du désir. À cet égard, elle suscite ce que nous appellerons le complexe de Joseph, ou d'Hippolyte (variante de celui d'(Edipe), qui impute à l'objet désiré un désir monstrueux pour l'innocent sujet masculin qui, s'il y cédait, se verrait instantanément dévoré. Plus il aime cette Phèdre, cette femme de Putiphar, voire cette Messaline ${ }^{9}$, plus il repousse la souillure du regard qu'elle jette sur lui. Mais la mère idéale est aussi un pôle identificatoire. Le narrateur ne semble pas trop heureux de ce que «le hasard» lui ait attribué la conduite de madame mère pendant la marche nocturne vers l'auberge (p. 321) alors que la fille, dont il ne se lasse pas de vanter les grâces, cheminait aux côtés d'un vieux bonhomme. Ce garçon manquait décidément d'adresse et de présence d'esprit. Mais, de toute façon, le dieu qui régit le sort s'appelle ici Marivaux (qu'on veuille bien tolérer l'anachronisme : l'auteur anonyme de la Voiture ne s'appellera Carlet de Marivaux qu'en 1716). Le hasard n'a rien à voir où la logique est si limpide. Certes, la dame est bien un peu ridicule avec sa façon de dramatiser la situation et de l'ennoblir par son geste et ses expressions, mais non moins frappante est la complicité du persifleur : «j'avais pénétré son caractère, vous pouvez vous imaginer que je m'y conformai et que je lui répondis d'un langage assortissant au sien» ; «j'entretenais, comme je vous ai dit, la dame d'un style tendre, merveilleux tout ensemble et grand». Telle était,

9 La passagère de la voiture embourbée a droit aux mêmes dénégations transparentes (p. 319) que la Félonde de Pharsamon. 
se vante-t-il, la «conformité dont j'usais avec ses idées». Un talent mimétique aussi brillant ne va pas de soi ; notre petit jeune homme n'aurait pu «user» avec autant de naturel de sa conformité avec les idées d'une dame mûrissante s'il ne les avait d'abord trouvées en lui. Viendrait-il par hasard d'écrire Les Effets surprenants de la sympathie ? Je $\mathrm{m}^{\prime}$ amusais à parler son langage, écrit-il, oubliant de noter qu'elle parle le sien.

D'ailleurs, où la belle voyageuse entre deux âges a-t-elle été chercher son style désuet et ampoulé ? Dans les romans de l'âge baroque qui perpétuent la tradition aristocratique et idéaliste des Amadis aimés de Don Quichotte. C'est en référence à cet univers-là qu'elle prend la défense de l'héroïsme chevaleresque en déclarant, par exemple,

que des impressions qui n'inspirent que des vertus ne devraient passer pour folles dans l'opinion de personne, et que les siècles passés ne les estimaient vertus que parce que la noblesse, la grandeur d'âme et le courage étaient parmi les hommes aussi ordinaires que le sont à présent l'intérêt, l'avarice et la volupté, qui ont insinué dans les sentiments des hommes un caractère petit et borné, qui ne ridiculise les antiques vertus que parce qu'elles ne sont pas ajustées à leur petitesse (p. 354-355).

Or ce sont là des paroles que l'on retrouve écrites plus ou moins textuellement en vingt endroits de l'œuvre de Marivaux comme l'expression de sa pensée à lui. On a de longtemps relevé aussi que la dame, dans sa partie du «Roman impromptu», inventera la donnée de La Double Inconstance, ce qui n'est pas si mal.

Bref, la fin de non-recevoir aigre et insistante qu'opposent tant de personnages de Marivaux, à l'instar du narrateur de La Voiture embourbée, à l'idée d'un rapprochement amoureux avec ce genre de femme, ne provient pas seulement de la carence de la triangulation odipienne. Dans un article intitulé «L'âge équivoque: Marivaux and the Middle-Aged Woman ${ }^{10}$ ", Janet Whatley écrit très bien à propos de cet auteur : «his great and lasting concern is how people construct ways of viewing themselves in order to love themselves» (p. 70). Or un puissant moyen d'acquérir une telle image valorisante de soi réside dans la relation avec un objet d'amour rayonnant. C'est l'impasse si la femme séduisante entre toutes est sur le point de dire adieu à la jeunesse, si donc elle se définit, comme c'était le cas à l'époque qui nous occupe, comme un être 
qui, faute d'exciter encore le désir, n'a plus rien qui intéresse personne. Que si d'autre part le sujet tente de sauver son amour en recourant à la stratégie qui engendre souvent l'homosexualité, c'est-à-dire en s'identifiant avec cette mère et en perpétuant leur relation dans un amour pour un garçon plus jeune qu'il aimera comme il a été aimé lui-même ou rêve de l'avoir été, ce chemin-là aussi est barré puisqu'il conduirait à se déplaire à soi-même en ressemblant à celle qui n'attire pas les hommes ${ }^{11}$.

Mais on a beau s'en défendre, les affinités profondes finissent toujours par triompher. Les conventions du roman héroïque, l'idéalisation de l'amour imputrescible parce qu'il s'interdit de sombrer dans l'abjection sous le fouet du plaisir, ce cocher sans merci, sont là pour garantir le maintien du lien avec l'Inaccessible, qu'on aime seule même si son incarnation sur la route de Nemours est un peu comique. C'est à elle que va la préférence. Le «hasard» a formé deux couples : le narrateur et la dame, le vieillard et la jeune demoiselle. Les premiers sont si profondément plongés dans la littérature qu'ils retombent sur l'un des plus beaux et des plus anciens de tous les romans. Rien ne les dérange dans leurs simagrées livresques même si, écrit le narrateur, «quelquefois l'eau des fossés nous surprenait jusqu'aux jambes» (p. 321). C'est ainsi qu'Iseut aux Blanches Mains révélait à son frère l'offensante chasteté de son mari, impuissant pour toute autre qu'Iseut la Blonde : "Cette eau, dont les sabots des chevaux m'ont éclaboussée sous ma robe, est plus hardie que ne le fut jamais Messire Tristan». Voilà ce qu'on appelle une entente parfaite. De l'autre côté, la demoiselle de quinze ans rit de tout son cœur à courir le cross-country pendant que le vieux rit aussi ; "par complaisance», certes, mais à l'entendre «de vingt pas [...] faire un hélan à chaque pied qu'il tirait de la boue» (p. 321 ; Deloffre explique en note que ces «hélans» sont des ahans), la pitié ne nous saisit pas nécessairement, car nous savons que bien des hommes sont prêts à se fatiguer au point de haleter pas mal s'ils en sont récompensés par la folle joie de leur partenaire.

11 Il va sans dire que ces dames entre deux âges ne sont qu'une illustration particulière de la difficulté à traiter avec la femme et le féminin. En plus de leur magnétisme érotique et sentimental, ces femmes se parent souvent d'attributs considérés comme masculins, qu'ils le soient en réalité, par tradition ou par métonymie : le pouvoir, l'argent, le sexe, le sabre, etc. À partir de là prolifèrent les élaborations de l'imaginaire. $C^{\prime}$ est ici pourtant que la clarté serait essentielle pour éviter de voir vaciller l'ordre que le symbolique a à faire prévaloir pour humaniser la différence biologique. Pousser plus loin dépasserait le cadre de la présente étude. - On lit avec profit Marivaux ou Le Dialogue avec la femme de Han Verhoeff, Orléans, Paradigme, 1994, 272 p. 
La formation de ces couples est d'autant plus remarquable que de nombreux traits rapprochent d'un autre côté le narrateur et la jeune fille. Autant il sympathise avec la mère dans son culte pour les amours à l'ancienne, autant il félicite l'autre de tourner le dos à l'imagination pour définir le bonheur comme l'union de l'amour et de la tendresse, c'est-àdire de la sensualité et du cœur (p. 319). Impossible de ne pas être d'accord, mais on sait trop bien que cette merveilleuse harmonie de l'âme et des sens, la plus désirable chose du monde, n'est certes pas la mieux partagée ; il n'est pas facile d'y atteindre, moins encore de la maintenir. Toujours est-il que, dans la fiction, l'auteur n'a pas osé s'y risquer en entreprenant la conquête de la demoiselle par le truchement de son représentant, le narrateur. Arrivé à l'auberge, celui-ci déplore que le vieillard soit encore à côté d'elle, «qui n'avait pu l'éviter» (p. 323); cela lui plaît à dire, mais il ne nous révèle pas par quels signes la victime de cette fâcheuse ordonnance de table a manifesté son déplaisir, ni d'ailleurs son contentement d'être assise à côté de lui, ce qui pourtant est également le cas. Il faut dire que le vieillard, ce sexagénaire dont on se complaît à nous répéter qu'il débite des compliments surannés et qu'il tousse et crache avant de se lancer dans son bout de roman, est néanmoins capable de siroter gaiement son vin toute la nuit après avoir franchi les marécages comme les autres (p. 335), que sa jeune voisine lui inspire une telle verve que la conversation finit par retomber sur l'amour (p. 323), qu'il sait où trouver de quoi manger et se propose pour l'aller quérir à onze heures du soir (p. 323-324), et que c'est lui qui trouve le moyen de se faire ouvrir la porte du curé (p. 329). Il n'est pas surprenant que le chapitre improvisé de ce prétendu croulant s'enchaîne le plus harmonieusement du monde à celui de la demoiselle : ce sont les deux seuls personnages du roman qui soient naturels, à l'aise et exempts de toute pose.

En revanche, ce qui ne se réalise pas dans la fiction a lieu dans l'écriture. Rubellin a relevé un nombre surprenant de traits qui font ressembler la jeune demoiselle au narrateur en tant que tel, et non plus en tant que personnage : formules identiques d'annonce et de conclusion, talent comique, recours à la métaphore du voyage pour parler du récit, mise en abyme de l'aventure de la voiture et de l'auberge dans la partie du roman impromptu dont elle est l'auteur, et jusqu'au terme «embourbée» qu'elle s'applique à elle-même comme narratrice ${ }^{12}$ ! Marivaux a encore fait d'elle son alliée sur le plan de la vie littéraire en lui 
confiant la délectable tâche de dégonfler la baudruche du bel esprit (p. 378-379).

Quant à celui-ci, il l'installe à deux reprises en porte-à-faux : il «faisait le coin", lit-on à un endroit, il s'assoit "sur le coin d'un banc» un peu plus bas (p. 323, 333). Effectivement, on ne le voit pas s'intéresser galamment à la mère ni à la fille. C'est un homme de trente-cinq ans,

qui parlait beaucoup, qui s'admirait à chaque fin de phrase, dont le geste brillait d'une vivacité plus présomptueuse que raisonnable, qui poussait la délicatesse jusqu'aux espaces imaginaires, qui la perdait de vue et la faisait perdre aux autres, et qui, malgré le néant sur lequel il parlait, trouvait le secret de ne point tarir son discours (p. 319-320).

Ce «néant» fait dresser l'oreille, parce qu'il rappelle une tirade célèbre de Pharsamon sur le Rien, ressort et moteur de tout, et en particulier origine de l'écriture ${ }^{13}$. Aussi bien, même sans la ressemblance entre les défauts que Marivaux souligne ici chez sa tête de Turc et ceux qu'on lui reprochera tant à lui-même, tout nous invite à considérer ce personnage comme le double et le repoussoir du narrateur. C'est un beau parleur qui fait de la littérature à propos de tout? Je le veux bien ; mais qui écrit, au moment où les voyageurs crottés arrivent à l'auberge : "notre hôtesse [...] ne savait que penser en nous voyant; si elle avait su la fable, peut-être nous eût-elle pris pour des immortels qui voyageaient» (p. 322) ? Si le bel esprit, «par un enthousiasme d'imagination né de la fatalité de notre situation, tâchait de nous dérober la fatigante attention que chacun de nous donnait à ses maux» (p. 321), en quoi cet enthousiasme diffère-t-il essentiellement de celui qui suggérera au narrateur-personnage l'idée du roman impromptu ? Et que fait le narrateur-écrivain, sinon transformer la réalité en prose, en plaisir esthétique la boue et la dégoûtante auberge que le lecteur affronte en imagination avec les voyageurs embourbés ? De fait, la tirade du bel esprit, résumant le discours burlesque et pathétique qu'il va tenir au curé pour l'attendrir (p. 325), est un amusant morceau et une parfaite mise en abyme du roman lui-même - à moins qu'on ne préfère la taxer de plagiat. En effet, ce personnage n'est pas méchant au sens où les méchants sont à craindre : il est trop ébloui de son propre scintillement pour tramer rien contre autrui ; mais les choses prendront une autre face si le narrateur n'est que 
le masque derrière lequel se cache un autre bel esprit, un auteur. Alors, évidemment, la rivalité se déclenche.

Et, s'ils sont tous deux de la même confrérie, nous ne nous étonnerons pas de leur voir partager un même trait de caractère, à savoir une certaine aigreur qui envenime les pointes, tordues et embarrassées mais non moins fielleuses, qu'ils décochent à leurs victimes. Le curé y goûte, par exemple, quand il s'excuse d'avoir laissé les voyageurs si longtemps à sa porte avant d'ouvrir : «Nous sommes trop bien traités, dit le bel esprit, pour des gens qui viennent demander des grâces l'argent à la main» (p. 330). Non seulement il fait comprendre au curé qu'il ne s'illusionne pas sur sa générosité, mais il le persifle : ou bien le bonhomme ne s'apercevra pas que le remerciement si élégamment tourné devient, du fait des derniers mots, une idiotie, ou bien il ne mérite pas mieux.

Dans ces cas-là, c'est d'argent qu'il s'agit. Le vieillard qui baguenaude si joyeusement avec la jeune fille, il est plus que temps de le rappeler, est un financier. «[...] le grand commerce qu'il avait avec l'argent lui donnait des idées communes, mais aisées et familières», lisons-nous (p. 320), et nous avons vu que ce n'étaient pas seulement ses idées qui étaient aisées, mais toute sa façon d'être. Cela est difficile à avaler pour les deux autres. La Voiture embourbée ne fait que des allusions détournées à la condition précaire des gens de lettres et à la nécessité où souvent ils sont réduits de faire la cour aux nantis. Ce n'est pas ici Le Neveu de Rameau, nous n'assistons pas aux empoignades de parasites que dépeindra Diderot. Pourtant, déjà ces passions que sont l'envie et l'amertume impuissante obscurcissent l'esprit. Par exemple, le narrateur s'obstine à interpréter défavorablement l'idée qu'a eue le bel esprit d'aller chercher des provisions chez le curé (p. 325, 332 ${ }^{14}$ ), alors que ce n'est pas lui qui y a pensé, mais le financier, et qu'il ne songe pas à usurper la paternité de cette initiative. Le rival est quand même impardonnable, car le projet, à défaut d'être le sien, lui a suggéré une improvisation virtuose. Intolérable également de voir ce rival revenir à l'auberge en triomphateur, avec du vin, du beurre et de la stockfiche, quand on était resté soi-même au chaud auprès des dames en se contentant de dénigrer une expédition déclarée futile a priori. Intolérable surtout ce retour victorieux quand on sait (l'auteur et le narrateur-écrivain le savent même si le narrateur-personnage ne peut pas le savoir, et la narratologie n'était pas encore venue

14 Dans le second de ces passages, l'algarade du narrateur n'est pas évidente à décoder. «La peste ! [...] la saillie qui vous a conduit chez ce curé n'est pas un coup de hasard, vous y excellez» s'interprète peut-être ainsi : Cher confrère, vous avez l'habitude de ces coups de génie : vous avez toujours brillé dans l'art de vous atteler au char de Plutus. 
mettre des bois dans les roues du ressentiment), intolérable, dis-je, quand on sait que lesdites victuailles furent arrachées à l'avarice du curé et de sa gouvernante non par le discours du bel esprit, «soutenu d'un écu qu'il tenait en main et dont on voyait bien qu'il allait payer ce qu'on lui donnerait» (p. 330), mais par la vertu de l'argent réellement donné et qui venait du financier.

Nous n'avons pas oublié que les deux suppliants étaient revenus du presbytère guidés par le neveu du curé. C'est un garçon «d'environ vingt-deux ans» (p. 330), donc le contemporain presque exact de Marivaux quand il écrivit La Voiture embourbée. L'éducation reçue dans son milieu campagnard a fait de lui un Pharsamon subalterne (aussi bien $n^{\prime}$ est-il pas noble), mais il ressemble aussi au valet Cliton $^{15}$ :

il avait assez bien fait ses études ; et malgré l'éducation champêtre qu'on lui avait donnée, au travers de la grossièreté qu'elle avait pu lui inspirer, on remarquait briller en lui une disposition d'esprit excellente que n'avait pu étouffer l'habitude de vivre avec des paysans ; entre autre chose, il avait lu des romans, et assez d'autres livres.

Autre double du narrateur, autre repoussoir. Mais, tandis que le bel esprit représentait la vacuité du beau parleur mondain, le neveu, lui, renvoie l'image déprimante du provincial qui débarque à Paris. Il ravive l'angoisse que dut éprouver certain étudiant en droit nommé Pierre Carlet quand il tomba de Riom chez ses oncles et cousins Bullet, architectes $\mathrm{du} \mathrm{Roi^{16 }}$. Le narrateur le prend sous son aile et le tourmente (p. 332-334). Il le pousse à se produire pour qu'on rie de lui ; il ne cesse de vanter l'esprit qu'on discernait sous ses dehors frustes, mais apporte en preuve une collection de sottises. Pourquoi le pauvre neveu est-il tellement plus mal traité que le Cliton de Pharsamon, lui aussi exhibé comme un rustre capable d'amuser la bonne compagnie, mais dont le récit fait ensuite l'objet d'une des plus magistrales apologies de notre littérature ${ }^{17}$ ? C'est qu'il a des prétentions à l'esprit. Malheur au rival qui fait rougir parce qu'on a risqué de lui ressembler, parce que certains, peut-être, trouvent qu' on lui ressemble encore. Marivaux lui retirera

15 Avec qui il partage le syndrome de la tête tournée (p. 332-333 ; voir Joly, "Pharsamon», p. 61-63).

16 Voir Michel Gilot, Les Journaux de Marivaux. Itinéraire moral et accomplissement esthétique, Paris, Champion, 1975, p. 23ss.

17 CEuvres de jeunesse, p. 602. 
presque entièrement l'occasion de faire ses preuves : il ne lui concède même pas quatre cents mots pour finir le roman avant que le cocher ne fasse remonter son monde en voiture.

En fin de compte, il y a quatre hommes dans l'auberge : d'un côté le financier et, de l'autre, les trois moins à l'aise, dans tous les sens du terme. Pourtant, dans l'esprit du narrateur, les choses se présentent autrement. Il imagine une complicité entre le vieillard et l'imposteur, qui brille sans être or ni l'avoir, qui profite de toutes les occasions pour étaler son strass, qui brandit son écu sans qu'on sache s'il en a tant que ça à donner ni s'il en donnera : bref, l'image même du succès creux. En face, le narrateur et son protégé, traité en alternance au fouet et aux caresses.

S'il n'arrive finalement rien au narrateur entre le début et la fin de $L a$ Voiture embourbée, c'est bien, j'espère l'avoir fait entrevoir, parce qu'il est paralysé tant au point de vue amoureux qu'au point de vue social. Il a du mal à entrer en relation avec autrui sur un autre mode que la dérision ou l'acrimonie, il ne sait pas ce qu'il est face à la femme et il hésite à se reconnaître dans les miroirs qui lui renvoient de l'homme de lettres l'image d'un beau parleur asexué ou d'un collégien balourd.

Mais qu'est-ce à dire? Quel est donc cet être ainsi conformé, quelle origine et quelle histoire l'ont fait tel ? Nulle autre origine que l'esprit de son auteur et nulle autre histoire que celle qui a mené cet auteur à écrire pareil roman. C'est pourquoi je ne m'étonne nullement de trouver chez ce personnage - dans ce qu'il est, fait et dit, dans ce qui lui arrive et aussi bien dans le livre tout entier - l'écho très net de préférences, de douleurs et d'impasses dont nous ne pouvons douter qu'elles furent celles de Marivaux. Car enfin, «Marivaux» et «l'auteur des œuvres de Marivaux» ne sont certes pas la même chose, puisque l'un est une personne historique concrète et l'autre, le sujet d'une énonciation ; mais il est absurde de se les représenter comme indépendants. Le second n'a pas d'autre corps pour prendre la parole que celui du premier, le premier doit une partie de son être au fait d'avoir été le lieu de la parole du second. C'est donc sans nous surprendre que nous voyons Marivaux, dans sa préface, brouiller la distinction entre un livre intitulé La Voiture embourbée et la lettre amicale qu'il est censé contenir. L'auteur du roman et le narrateur censé être allé à Nemours ne sont ni plus ni moins réels ou fictifs l'un que l'autre, dans la mesure où «moi, l'auteur» serait une fiction s'il prétendait coüncider avec l'intégralité et les limites de sa personne historique concrète, et puisque le narrateur est réel d'être une production mentale réelle de ladite personne historique concrète.

Mais on ne passe pas d'un statut à l'autre sans de multiples médiations. Certaines sont modulées par la tradition et les particularités du 
milieu culturel. C'est dans cette optique, qui relève de l'histoire de la littérature au sens le plus noble, qu'a travaillé Jean-Paul Sermain pour produire son important ouvrage sur les romans parodiques de Marivaux $^{18}$. À propos de La Voiture embourbée, il montre comment l'une de ses fonctions essentielles est de contraindre à s'interroger sur la position de l'écrivain dans le tissu social, sur l'étendue et la forme de la maîtrise et de la liberté qu'il conquiert, face aux autres que représentent par exemple ici les compagnons de voyage du narrateur, grâce à "des manières différentes de pratiquer le langage, et d'en maîtriser littérairement les usages» (p. 140). C'est ainsi que l'écrivain parvient «à donner à son existence une forme, à manifester un style propre. Face au vide» (p. 144).

Les pages qu'on vient de lire débouchent sur un autre vide - où est-ce le même, approché d'un autre angle ? - et sur la littérature comme moyen de n'y pas sombrer. Il ne faudrait pas s'imaginer qu'on choisit le «sujet» de son écriture. Ce qui fait écrire domine au contraire l'écrivain et lui est imposé doublement, comme unique et comme général : chacun est pris dans les particularités de son histoire, qui déterminent la forme sous laquelle il vivra l'universel, à savoir l'irrémédiable insuffisance du monde devant les contradictions qui ravagent le sujet humain. $\mathrm{Si}$, dans l'acte transformateur de la production du texte, l'écrivain acquiert de la liberté et de la maîtrise, c'est d'avoir mis entre parenthèses la recherche $\mathrm{d}^{\prime}$ une satisfaction réelle propre à colmater les brèches de son être. Je ne sais pas, et j'ignore si quelqu'un sait, à quelle économie particulière du désir il doit d'opter pour cette singulière pratique qu'est la littérature, pourquoi il choisit de consacrer tant de veilles à une parole qui ne sert à rien. Il tient cependant à l'adresser à la phalange de tous ceux qui peuvent l'entendre, comme si l'impasse de son désir, peu importe qu'il en étale plus ou moins crûment la radicale subjectivité, était devenue secondaire par rapport à la postulation d'une réponse sur un autre théâtre que celui, trivial, de sa vie et de sa mort.

RAYMOND JOLY

Université Laval

18 Le Singe de Don Quichotte: Marivaux, Cervantes et le roman postcritique, Oxford, Voltaire Foundation, Studies on Voltaire and the Eighteenth Century, 368, 1999. 